\title{
Relationship between progression of visual field defect and intraocular pressure in primary open-angle glaucoma
}

This article was published in the following Dove Press journal:

Clinical Ophthalmology

23 July 2015

Number of times this article has been viewed

\author{
Tomoko Naito' \\ Keiji Yoshikawa ${ }^{2}$ \\ Shiro Mizoue ${ }^{3}$ \\ Mami Nanno ${ }^{4}$ \\ Tairo Kimura ${ }^{5}$ \\ Hirotaka Suzumura ${ }^{6}$ \\ Fumio Shiraga' \\ 'Department of Ophthalmology, \\ Okayama University Graduate \\ School of Medicine, Okayama, Japan; \\ ${ }^{2}$ Yoshikawa Eye Clinic, Tokyo, Japan; \\ ${ }^{3}$ Department of Ophthalmology, \\ Ehime University Graduate School \\ of Medicine, Ehime, Japan; ${ }^{4}$ Kagurazaka \\ Minamino Eye Clinic, ${ }^{5}$ Ueno Eye \\ Clinic, ${ }^{6}$ Suzumura Eye Clinic, \\ Tokyo, Japan
}

Correspondence: Tomoko Naito Department of Ophthalmology, Okayama University Graduate School of Medicine, 2-5-I Shikata-cho, Kita-ku, Okayama 700-8558, Japan

Tel +8I 862357297

Fax +8I 862225059

Email tomokoum@gmail.com
Purpose: To analyze the relationship between intraocular pressure (IOP) and the progression of visual field defects in Japanese primary open-angle glaucoma (POAG) and normal-tension glaucoma (NTG) patients.

Patients and methods: The subjects of the study were patients undergoing treatment for POAG or NTG who had performed visual field tests at least ten times with a Humphrey field analyzer (Swedish interactive thresholding algorithm standard, C30-2 program). The progression of visual field defects was defined by a significantly negative value of the mean deviation slope at the final visual field test during the follow-up period. The relationships between the progression of visual field defects and IOP, as well as other clinical factors, were retrospectively analyzed.

Results: A total of 156 eyes of 156 patients were included in the analysis. Significant progression of visual field defects was observed in 70 eyes of 70 patients (44.9\%), while no significant progression was evident in 86 eyes of 86 patients $(55.1 \%)$. The eyes with visual field defect progression had significantly lower baseline IOP $(P<0.05)$, as well as significantly lower IOP reduction rate $(P<0.01)$. The standard deviation of IOP values during follow-up was significantly greater in the eyes with visual field defect progression than in eyes without $(P<0.05)$.

Conclusion: Reducing IOP is thought to be useful for Japanese POAG or NTG patients to suppress the progression of visual field defects. In NTG, IOP management should take into account not only achieving the target IOP, but also minimizing the fluctuation of IOP during follow-up period.

Keywords: primary open-angle glaucoma, normal-tension glaucoma, intraocular pressure, progression of visual field defects, MD slope

\section{Introduction}

The estimated prevalence of normal-tension glaucoma (NTG) in Japan is high, at $3.6 \%$, accounting for approximately $90 \%$ of cases of primary open-angle glaucoma (POAG) (estimated prevalence 3.9\%) and approximately $70 \%$ of all cases of glaucoma $(5.0 \%) .{ }^{1,2} \mathrm{With}$ recent advances in the understanding of glaucoma pathology, NTG, in which intraocular pressure (IOP) remains within the normal range, has been recognized to be a pathology of POAG associated with IOP that exceeds the statistically normal value of $21 \mathrm{mmHg}$.

Since the difference between POAG and NTG is thought to be the individual difference in the vulnerability of the optic disc to increase in IOP in the process of the development and progression of glaucomatous optic neuropathy, it is difficult to distinguish in practice between POAG and NTG at a specific level of IOP.

At present, the only evidence-based remedy for glaucoma is to lower IOP. ${ }^{3-7}$ The practical goal of treatment is to reduce IOP by $20 \%-30 \%$ from baseline. ${ }^{3-5,8-12}$ The association 
between a reduction in IOP and the progression of visual field defects has been fully studied for POAG. Relatively high IOP has also been recognized to be evaluated as a major risk factor for the progression of visual field defects in NTG. ${ }^{4,5,13,14}$ NTG is, therefore, treated in the same way as POAG, although there have been few large-scale studies that had as their main focus on IOP and the progression of visual field defects.

It was, therefore, decided to analyze the relationship between IOP and the progression of visual field defects in Japanese POAG and NTG patients.

\section{Materials and methods}

\section{Study design}

This was a multicenter, retrospective, and observational study.

\section{Subjects}

The study subjects were patients who underwent treatment for POAG or NTG between 1999 and 2011 at Okayama University, Minami-Matsuyama Hospital, Kagurazaka Minamino Eye Clinic, Ueno Eye Clinic, Suzumura Eye Clinic, Yoshikawa Eye Clinic, and Nihonmatsu Eye Hospital who met the enrolment conditions and did not fulfill the exclusion criteria described below.

The inclusion criteria for this study were: 1 ) age $\geq 20$ years; 2) having obvious glaucomatous visual field defects in accordance with Anderson's criteria; ${ }^{15} 3$ ) having visual field testing with a Humphrey field analyzer (Zeiss-Humphrey Systems, San Leandro, CA, USA) at least a total of ten times, in principle every 6 months without alteration in strategy (Swedish interactive thresholding algorithm standard) or program (C30-2); 4) capable of undergoing assessment of the mean deviation (MD) slope by using HfaFiles (Beeline, Tokyo, Japan); 5) baseline IOP was measured at least three times applying a Goldmann applanation tonometer before undergoing treatment; and 6) IOP was measured regularly during the follow-up period.

The exclusion criteria were as follows: 1) $\mathrm{MD}<-20 \mathrm{~dB}$ on initial visual field testing; 2) corrected visual acuity during follow-up $<0.7 ; 3$ ) any catch trials of visual field testing (fixation loss, false positives, and false negatives) $\geq 33 \%$; 4) having an ophthalmic disorder other than glaucoma that might affect the visual field; 5) having undergone ophthalmic surgery (including intraocular lens implantation) during the follow-up period; 6) having abnormalities of the anterior segment that might disturb accurate Goldmann applanation tonometer measurements; 7) history of recurrent uveitis, scleritis, or corneal herpes; 8) pregnancy or lactation; 9) patients with severe dementia or serious ophthalmic conditions; or 10) patients judged by an investigator to be ineligible to participate in this study.

Sex was not considered, and there were no restrictions on the type of eye drops used as treatment by patients.

\section{Procedure}

The MD and MD slope were calculated using HfaFiles. If the MD slope had a significant negative value at the time of final visual field testing during the follow-up period, the visual field defect was defined as having progressed.

Baseline IOP was defined by calculating the mean value of three IOP measurements before glaucoma treatment. The mean of all IOP values measured during the follow-up period after the start of treatment was regarded as the mean IOP in this study. The difference between baseline IOP and mean IOP was defined as $\triangle \mathrm{IOP}$, and the proportion of $\triangle \mathrm{IOP}$ of baseline IOP was taken as the reduction rate of IOP. The standard deviation of the mean follow-up IOP was also calculated. In the present study, the mean of the highest and second-highest IOP, as well as the lowest and second-lowest IOP, was defined as the maximum IOP and the minimum IOP, respectively.

\section{Statistical analysis}

The protocol for this study was approved in advance by the Institutional Review Board of Nihonmatsu Eye Hospital. Informed consent was obtained from the subjects after a thorough explanation of objective of the study and information collection was given in accordance with ethical principles based on the Helsinki Declaration. Test data were collected separately by each participating facility.

One eye from each subject who met the selection criteria and did not fulfill the exclusion criteria was selected for the analysis. If both eyes met the selection criteria, the eye with the lower initial MD value was chosen. Data analysis was performed at Okayama University. A $t$-test or Fisher's exact test was used for the analysis of each factor, depending on the data scale concerned. IOP related and background factors potentially associated with the occurrence of MD slope worsening were adopted for multiple logistic regression analysis. All variables were selected as non-multicollinearity after employment of variables' correlation coefficient less than 0.3. The analytical software used was JMP Version 9.0 (SAS Institute Inc., Cary, NC, USA), and the level of significance was set at $5 \%$ on both sides.

\section{Results}

A total of 156 eyes of 156 patients comprised 94 females $(60.3 \%)$ and 62 males $(39.7 \%)$ with the age of 
$64.6 \pm 12.9$ years (mean \pm standard deviation) were served for the analysis. The type of disease was NTG in 141 patients (90.4\%) and POAG in 15 (9.6\%). Refraction was $-2.8 \mathrm{D} \pm 3.7 \mathrm{D}$, baseline IOP was $16.6 \pm 3.7 \mathrm{mmHg}$, initial MD was $-7.08 \pm 4.21 \mathrm{~dB}$, final MD was $-9.42 \pm 5.32 \mathrm{~dB}$, the number of visual field tests was $12.8 \pm 2.7$ times, and the follow-up period was $7.6 \pm 2.0$ years (Table 1).

Significant deterioration of visual field defects was observed in 70 eyes of 70 patients (MD slope worsening group, $44.9 \%$ ), while no significant deterioration was evident in 86 eyes of 86 patients (55.1\%) (MD slope non-worsening group). In the MD slope worsening group, age (66.9 vs 62.6 years, $P=0.0370)$, proportion of female $(P=0.0324)$, and degree of refraction $(-1.9 \mathrm{D}$ vs $-3.5 \mathrm{D}, P=0.0048)$ were significantly higher as compared to the MD slope non-worsening group. However, there were no significant differences between the two groups in terms of initial MD value ( $-6.60 \mathrm{~dB}$ vs $-7.48 \mathrm{~dB}, P=0.1916)$.

A comparison of IOP between worsening and nonworsening group revealed no obvious differences in mean IOP (13.2 mmHg vs $13.5 \mathrm{mmHg}, P=0.3992$ ) or maximum IOP (16.9 mmHg vs $16.7 \mathrm{mmHg}, P=0.8147$ ) during follow-up, but there were significant differences between the two groups in terms of baseline IOP $(15.7 \mathrm{mmHg}$ vs $17.2 \mathrm{mmHg}, P=0.0107)$, reduction rate of IOP during follow-up $(14.9 \%$ vs $20.3 \%, P=0.0066)$, minimum IOP (10.2 $\mathrm{mmHg}$ vs $11.0 \mathrm{mmHg}, P=0.0161$ ), and the standard

Table I Patients' characteristics

\begin{tabular}{ll}
\hline Number of subjects & I56 eyes of I56 patients \\
Age (years) & $64.6 \pm 12.9[28-88]$ \\
Sex, $\mathrm{n}(\%)$ & $94(60.3)$ \\
$\quad$ Female & $62(39.7)$ \\
$\quad$ Male & \\
Type of glaucoma, $\mathrm{n}(\%)$ & $141(90.4)$ \\
$\quad$ NTG & $15(9.6)$ \\
$\quad$ POAG & $-2.8 \pm 3.7[-16$ to +2.5$]$ \\
Refraction (D) & $16.6 \pm 3.7[8.0-28.6]$ \\
Baseline IOP (mmHg) & $-7.08 \pm 4.21[-18.39$ to \\
Initial MD value (dB) & $+0.03]$ \\
& $-9.42 \pm 5.32[-25.49$ to \\
Final MD value (dB) & $+0.67]$ \\
& $12.8 \pm 2.7[10-25]$ \\
Number of visual field & \\
examinations (times) & $7.6 \pm 2.0[4.5-16.5]$ \\
Follow-up duration (years) &
\end{tabular}

Notes: Data are $\mathrm{n}(\%)$ or means $\pm \mathrm{SD}$ [range]. NTG defined as baseline $\mathrm{IOP} \leq 2 \mathrm{I} \mathrm{mmH}$; POAG defined as baseline IOP $>21 \mathrm{mmHg}$; baseline IOP determined by averaging first three measurements taken with Goldmann applanation tonometer before initiation of therapy.

Abbreviations: IOP, intraocular pressure; MD, mean deviation; NTG, normaltension glaucoma; POAG, primary open-angle glaucoma; SD, standard deviation. deviation of IOP during the follow-up period (1.7 $\mathrm{mmHg}$ vs $1.4 \mathrm{mmHg}, P=0.0309$ ).

The fluctuations in IOP are smaller for patients with NTG than for those with POAG, both for patients with worsening of the MD slope (NTG: $1.7 \pm 0.9 \mathrm{mmHg}$ vs POAG: $2.2 \pm 0.7 \mathrm{mmHg}$ ) and for those in whom no such worsening was evident (NTG: $1.4 \pm 0.5 \mathrm{mmHg}$ vs POAG: $1.7 \pm 0.5 \mathrm{mmHg}$ ) (Table 2).

Multiple logistic regression analysis was applied IOP value over follow-up, IOP reduction rate, long-term IOP fluctuation, initial MD value, and refraction that was not affected by multicollinearity, as the objective variable against the presence/absence of MD slope worsening. The results showed significant association between IOP reduction rate (odds ratio [OR]: 0.97, 95\% confidence interval [CI]: 0.94-0.99, $P<0.05$ ), long-term IOP fluctuation (OR: 1.87, 95\% CI: 1.13-3.38, $P<0.05$ ), refraction (OR: 1.12 , 95\% CI: $1.01-1.24, P<0.05)$, and the presence of MD slope worsening (Table 3).

\section{Discussion}

In Japanese POAG and NTG eyes, decrease in IOP was appeared to be evident in the eyes without significant visual filed progression as compared to those eyes with significant visual field worsening during follow-up period of $7.6 \pm 2.0$ years.

At present, the only evidence-based remedy for POAG and NTG in which IOP does not exceed normal limits is almost the same, ie, to lower IOP from the baseline levels. Although NTG is the most common glaucoma type in Japan, few studies have focused on the relationship between the deterioration of visual field defects and the levels of IOP under medical treatment. ${ }^{14}$ In this study, therefore, the effect of IOP reduction on visual field defect deterioration in POAG and NTG eyes was retrospectively evaluated in a multicenter study including one university hospital and six eye clinics. There are eye drops that have been reported to have neuroprotective effects such as brimonidine. ${ }^{16}$ But brimonidine was approved in 2012 in Japan, we could not prescribe brimonidine at the time of analysis of this study. Purpose of the present study is to analyze the relationship between IOP and the progression of visual field defects. Thus, the subjects were neither restricted by the type of eye drops in the inclusion criteria nor performed analysis by each eye drops.

Among the variety of parameters used to determine the progression of visual field defects, MD slope is thought to be one of the most standard and commonly used index for analyzing the course of glaucomatous visual field defects. Some participating facilities in this study had the Humphrey 
Table 2 Associations between different factors and worsening of the MD slope

\begin{tabular}{|c|c|c|c|}
\hline & \multicolumn{2}{|l|}{ MD slope } & \multirow[t]{2}{*}{$P$-value ${ }^{a}$} \\
\hline & Worsening group $(n=70)$ & Non-worsening group $(n=86)$ & \\
\hline Age (years) & $66.9 \pm 11.3$ & $62.6 \pm 13.9$ & $0.0370 *$ \\
\hline \multicolumn{4}{|l|}{ Sex, n (\%) } \\
\hline Female & $49(70.0)$ & $45(52.3)$ & $0.0324 *$ \\
\hline Male & $21(30.0)$ & 4I (47.7) & \\
\hline Refraction (D) & $-1.9 \pm 3.8$ & $-3.5 \pm 3.4$ & $0.0048 *$ \\
\hline Initial MD value $(\mathrm{dB})$ & $-6.60 \pm 4.17$ & $-7.48 \pm 4.23$ & 0.1916 \\
\hline MD slope (dB/year) & $-0.7 I \pm 0.45$ & $-0.0 \mathrm{I} \pm 0.28$ & $<0.000 I^{*}$ \\
\hline Baseline IOP (mmHg) & $15.7 \pm 3.6$ & $17.2 \pm 3.6$ & $0.0107^{*}$ \\
\hline IOP over follow-up (mmHg) & $13.2 \pm 2.2$ & $13.5 \pm 2.1$ & 0.3992 \\
\hline IOP reduction rate $(\%)$ & $\mid 4.9 \pm 13.0$ & $20.3 \pm 11.8$ & $0.0066 *$ \\
\hline Maximum IOP $(\mathrm{mmHg})$ & $16.9 \pm 3.8$ & $16.7 \pm 3.0$ & 0.8147 \\
\hline Minimum IOP (mmHg) & $10.2 \pm 2.1$ & $11.0 \pm 2.0$ & $0.0161 *$ \\
\hline Long-term IOP fluctuation $(\mathrm{mmHg})$ & $1.7 \pm 0.9$ & $1.4 \pm 0.5$ & $0.0309 *$ \\
\hline
\end{tabular}

Notes: Data are means \pm SD or $n(\%)$. Long-term IOP fluctuation was calculated as the SD of IOP measurements over the follow-up period. ${ }^{a}$-test except sex (Fisher's exact test). *Statistically significant.

Abbreviations: IOP, intraocular pressure; MD, mean deviation; SD, standard deviation.

Visual Field Analyzer models that had not been equipped visual field index. MD slope was, therefore, applied as an index of visual field progression in the present study. In addition, we excluded patients with corrected visual acuity during follow-up was less than 0.7 by the exclusion criteria, thus the effect of cataract was considered to be relatively minor. A significantly negative value of the MD slope at the final visual field test was defined as apparent visual field deterioration in each eye in the present study.

Of all eyes analyzed, the majority were NTG eyes $(90.4 \%$, $141 / 156)$ in this study, reflecting the frequency of that glaucoma type in Japan. ${ }^{1,2}$ The baseline IOP of the present subjects was $16.6 \pm 3.7 \mathrm{mmHg}$, but the mean IOP during follow-up was $13.3 \pm 2.2 \mathrm{mmHg}$.

In the present study, mean age was significantly higher in the MD slope worsening group $(P=0.0370)$. This result came in line with those of the Early Manifest Glaucoma Trial ${ }^{9}$ and the Canadian Glaucoma Study Group. ${ }^{17}$ The Tajimi study reported that there was no significant difference in NTG

Table 3 Factors related to the MD slope worsening according to multivariate logistic regression analysis $(n=\mid 56)$

\begin{tabular}{llll}
\hline Factors & MD slope & \multicolumn{1}{l}{$P$-value } \\
\cline { 2 - 3 } & Odds ratio & $\mathbf{9 5 \%} \mathbf{~ C l}$ & \\
\hline IOP over follow-up (mmHg) & 0.89 & $0.75-1.04$ & 0.1421 \\
IOP reduction rate (\%) & 0.97 & $0.94-0.99$ & $0.0250^{*}$ \\
Long-term IOP fluctuation (mmHg) & 1.87 & $1.13-3.38$ & $0.0143^{*}$ \\
Initial MD value (dB) & 1.05 & $0.97-1.15$ & 0.2536 \\
Refraction (D) & 1.12 & $1.01-1.24$ & $0.0285^{*}$ \\
\hline
\end{tabular}

Notes: Long-term IOP fluctuation was calculated as the SD of IOP measurements over the follow-up period. *Statistically significant.

Abbreviations: $\mathrm{Cl}$, confidence interval; IOP, intraocular pressure; MD, mean deviation; $\mathrm{SD}$, standard deviation. frequency between males and females. ${ }^{1}$ However, in the present study, a significantly higher frequency of apparent progression of visual field defects was observed in females than in males $(P=0.0324)$. Similar results have also been reported by Collaborative Normal-Tension Glaucoma Study ${ }^{18}$ and the Canadian Glaucoma Study Group. ${ }^{17}$ Early menopause is significantly associated with the prevalence of glaucoma, and it has been suggested that endogenous estrogen can have a protective effect against glaucoma. ${ }^{19,20}$ Estrogen replacement therapy has also been shown to reduce the resistance of the ophthalmic artery, ${ }^{21,22}$ as well as plasma viscosity. ${ }^{22}$ However, the effect of estrogen on the progression of visual field defects remains unclear, and further study is needed. Refraction was also significantly higher among patients with apparent visual field deterioration $(-1.9 \mathrm{D}$ vs $-3.5 \mathrm{D}$, $P=0.0048)$. Though the Tajimi study reported that the severity of myopia correlated with the onset rate of glaucoma, ${ }^{1,2}$ it is still unclear if there was a relationship between the severity of myopia and the progression of visual field defects. ${ }^{23-25}$ Further investigation may also be required for this issue.

In the treatment of NTG eyes, reduction of IOP is mandatory, as in POAG eyes. A decrease of $1-2 \mathrm{mmHg}$ is believed to be a valuable therapeutic effect. ${ }^{26}$ In the present study, therefore, the main focus was on various IOP-derived factors such as the IOP reduction rate, the standard deviation of IOP during the follow-up period, and the maximum and minimum values to assess the effect of IOP reduction on visual field deterioration.

There was no significant difference in the mean IOP during follow-up between the eyes with visual field deterioration and those without (13.2 $\mathrm{mmHg}$ vs $13.5 \mathrm{mmHg}, P=0.3992)$, but the 
IOP reduction rate was significantly lower ( $14.9 \%$ vs $20.3 \%$, $P=0.0066$ ) in the eyes with significant visual field deterioration as compared to those without significant deterioration.

Conversely, however, the baseline IOP was significantly lower in the eyes with visual field deterioration compared to those eyes without (15.7 $\mathrm{mmHg}$ vs $17.2 \mathrm{mmHg}, P=0.0107$ ), and minimum IOP was also significantly lower $(10.2 \mathrm{mmHg}$ vs $11.0 \mathrm{mmHg}, P=0.0161)$. These results suggest that, as the first step, target IOP should be set at a reduction of at least $20 \%$ from baseline IOP for NTG eyes, as in POAG eyes. It is also suggested that the IOP reduction rate is a more sensitive indicator to assess the sufficiency of IOP reduction during the follow-up period in POAG/NTG eyes.

The Collaborative Initial Glaucoma Treatment Study ${ }^{27}$ as well as Advanced Glaucoma Intervention Study ${ }^{28}$ reported that fluctuations in IOP during follow-up were apparently related to the progression of visual field defects. Decrease in risk of developing glaucoma was evident in cases with $20 \%$ reduction of IOP in Ocular Hypertension Treatment Study. ${ }^{29}$ In this study, the non-worsening group achieved $20.3 \%$ reduction of IOP, had smaller IOP fluctuation compared with worsening group, and multiple logistic regression suggested the influence of IOP reduction rate and IOP fluctuation in the progression of visual field defects. According to these results, it was noted that $20 \%$ reduction of IOP and minimizing of IOP fluctuation during follow-up may be effective for POAG/NTG eyes to slowdown further worsening of visual field defects.

In this study, the standard deviation of IOP during follow-up was thought to reflect long-term IOP fluctuation, and it was also significantly greater in eyes with MD slope worsening than in those without worsening $(1.7 \mathrm{mmHg}$ vs $1.4 \mathrm{mmHg}, P=0.0309)$. IOP fluctuates on a diurnal, day-today, and seasonal basis. ${ }^{30-33}$ Since this was a multicenter, retrospective study, it was difficult to set the timeframe of IOP measurement, and this was a limitation of the study. However, the standard deviation of the IOP among POAG eyes without visual field progression was $1.7 \mathrm{mmHg}$, less than the $2 \mathrm{mmHg}$ that is generally regarded as the normally observed diurnal or day-to-day fluctuation. The mean value was $2.2 \mathrm{mmHg}$ for POAG eyes with MD slope worsening, suggesting that fluctuations in IOP that exceed $2 \mathrm{mmHg}$ are to some degree related to the deterioration of visual field defects. Though the standard deviation in IOP among NTG eyes was within $2 \mathrm{mmHg}$ for both eyes with $(1.7 \mathrm{mmHg}$ ) or without (1.4 $\mathrm{mmHg}) \mathrm{MD}$ slope worsening, the standard deviation was significantly greater $(P=0.0320)$ in MD slope worsening eyes. It is, therefore, postulated that an increase in the standard deviation of IOP during follow-up may be a risk factor for the progression of visual field defects, particularly in NTG eyes. According to Caprioli, ${ }^{34}$ longterm fluctuations in IOP may cause the breakdown of the homeostasis necessary for the protection of retinal ganglion cells. Stabilization of fluctuating IOP during follow-up may be necessary for NTG eyes to slowdown further worsening of visual field defects.

The results of this study reconfirmed that, for POAG/NTG treatment, as the first step, the target IOP should be set as at least a $20 \%$ reduction relative to the baseline IOP. It was also thought to be important, to confirm IOP reduction rate and IOP fluctuations on a regular basis, particularly in NTG eyes, during the follow-up period.

\section{Disclosure}

The authors report no conflicts of interest in this work.

\section{References}

1. Iwase A, Suzuki Y, Araie M, et al. The prevalence of primary open-angle glaucoma in Japanese: the Tajimi study. Ophthalmology. 2004;111(9): 1641-1648.

2. Yamamoto T, Iwase A, Araie M, et al. The Tajimi study report 2: prevalence of primary angle closure and secondary glaucoma in a Japanese population. Ophthalmology. 2005;112(10):1661-1669.

3. [The Japan Glaucoma Society Guidelines for Glaucoma (3rd Edition)] Nihon Ganka Gakkai Zasshi. 2012;116(1):3-46. Japanese.

4. Collaborative Normal-Tension Glaucoma Study Group. Comparison of glaucomatous progression between untreated patients with normaltension glaucoma and patients with therapeutically reduced intraocular pressures. Am J Ophthalmol. 1998;126(4):487-497.

5. Collaborative Normal-Tension Glaucoma Study Group. The effectiveness of intraocular pressure reduction in the treatment of normal-tension glaucoma. Am J Ophthalmol. 1998;126(4):498-505.

6. European Glaucoma Society. Terminology and Guidelines for Glaucoma. 3rd ed. Italy: European Glaucoma Society; 2008.

7. Canadian Ophthalmological Society Glaucoma Clinical Practice Guideline Expert Committee; Canadian Ophthalmological Society. Canadian Ophthalmological Society evidence-based clinical practice guidelines for the management of glaucoma in the adult eye. Can J Ophthalmol. 2009;44 Suppl 1:S7-S93.

8. Heijl A, Leske MC, Bengtsson B, Hyman L, Bengtsson B, Hussein M; Early Manifest Glaucoma Trial Group. Reduction of intraocular pressure and glaucoma progression: results from the Early Manifest Glaucoma Trial. Arch Ophthalmol. 2002;120(10):1268-1279.

9. Leske MC, Heijl A, Hussein M, Bengtsson B, Hyman L, Komaroff E; Early Manifest Glaucoma Trial Group. Factors for glaucoma progression and the effect of treatment: the early manifest glaucoma trial. Arch Ophthalmol. 2003;121(1):48-56.

10. Kass MA, Heuer DK, Higginbotham EJ, et al. The Ocular Hypertension Treatment Study: a randomized trial determines that topical ocular hypotensive medication delays or prevents the onset of primary openangle glaucoma. Arch Ophthalmol. 2002;120(6):701-713.

11. Miglior S, Zeyen T, Pfeiffer N, Cunha-Vaz J, Torri V, Adamsons I; European Glaucoma Prevention Study (EGPS) Group. Results of the European Glaucoma Prevention Study. Ophthalmology. 2005;112(3): 366-375.

12. AGIS Investigators. The Advanced Glaucoma Intervention Study (AGIS): 7. The relationship between control of intraocular pressure and visual field deterioration. Am J Ophthalmol. 2000;130(4):429-440. 
13. Cartwright MJ, Anderson DR. Correlation of asymmetric damage with asymmetric intraocular pressure in normal-tension glaucoma (lowtension glaucoma). Arch Ophthalmol. 1988;106(7):898-900.

14. Araie M, Sekine M, Suzuki Y, Koseki N. Factors contributing to the progression of visual field damage in eyes with normal-tension glaucoma. Ophthalmology. 1994;101(8):1440-1444.

15. Anderson DR, Patella VM. Automated Static Perimetry. 2nd ed. St Louis (MO): Mosby; 1999:121-190.

16. Galanopoulos A, Goldberg I. Clinical efficacy and neuroprotective effects of brimonidine in the management of glaucoma and ocular hypertension. Clin Ophthalmol. 2009;3:117-122.

17. Chauhan BC, Mikelberg FS, Balaszi AG, LeBlanc RP,LeskMR, Trope GE; Canadian Glaucoma Study Group. Canadian Glaucoma Study: 2. Risk factors for the progression of open-angle glaucoma. Arch Ophthalmol. 2008;126(8):1030-1036.

18. Drance S, Anderson DR, Schulzer M; Collaborative Normal-Tension Glaucoma Study Group. Risk factors for progression of visual field abnormalities in normal-tension glaucoma. Am J Ophthalmol. 2001;131(6): 699-708.

19. Hulsman CA, Westendorp IC, Ramrattan RS, et al. Is open-angle glaucoma associated with early menopause? The Rotterdam Study. Am J Epidemiol. 2001;154(2):138-144.

20. Lee AJ, Mitchell P, Rochtchina E, Healey PR; Blue Mountains Eye Study. Female reproductive factors and open angle glaucoma: the Blue Mountains Eye Study. Br J Ophthalmol. 2003;87(11):1324-1328.

21. Harris-Yitzhak M, Harris A, Ben-Refael Z, Zarfati D, Garzozi HJ, Martin BJ. Estrogen-replacement therapy: effects on retrobulbar hemodynamics. Am J Ophthalmol. 2000;129(5):623-628.

22. Battaglia C, Mancini F, Regnani G, Persico N, Volpe A, De Aloysio D. Hormone therapy and ophthalmic artery blood flow changes in women with primary open-angle glaucoma. Menopause. 2004;11(1):69-77.

23. Chihara E, Liu X, Dong J, et al. Severe myopia as a risk factor for progressive visual field loss in primary open-angle glaucoma. Ophthalmologica. 1997;211(2):66-71.
24. Araie M, Shirato S, Yamazaki Y, Matsumoto C, Kitazawa Y, Ohashi Y; Nipradilol-Timolol Study Group. Risk factors for progression of normal-tension glaucoma under $\beta$-blocker monotherapy. Acta Ophthalmol. 2012;90(5):e337-e343.

25. Phelps CD. Effect of myopia on prognosis in treated primary open-angle glaucoma. Am J Ophthalmol. 1982;93(5):622-628.

26. Tomita G, Araie M, Kitazawa Y, Tsukahara S. A three-year prospective, randomized and open comparison between latanoprost and timolol in Japanese normal-tension glaucoma patients. Eye (Lond). 2004;18(10): 984-989.

27. Musch DC, Gillespie BW, Niziol LM, Lichter PR, Varma R; CIGTS Study Group. Intraocular pressure control and long-term visual field loss in the Collaborative Initial Glaucoma Treatment Study. Ophthalmology. 2011;118(9):1766-1773.

28. Caprioli J, Coleman AL. Intraocular pressure fluctuation a risk factor for visual field progression at low intraocular pressures in the advanced glaucoma intervention study. Ophthalmology. 2008;115(7):1123-1129.

29. Medeiros FA, Weinreb RN, Zangwill LM, et al. Long-term intraocular pressure fluctuations and risk of conversion from ocular hypertension to glaucoma. Ophthalmology. 2008;115(6):934-940.

30. Blumenthal M, Blumenthal R, Peritz E, Best M. Seasonal variation in intraocular pressure. Am J Ophthalmol. 1970;69(4):608-610.

31. Kitazawa Y, Horie T. Diurnal variation of intraocular pressure in primary open-angle glaucoma. Am J Ophthalmol. 1975;79(4):557-566.

32. Ido T, Tomita G, Kitazawa Y. Diurnal variation of intraocular pressure of normal-tension glaucoma. Influence of sleep and arousal. Ophthalmology. 1991;98(3):296-300.

33. Wilensky JT. Diurnal variations in intraocular pressure. Trans Am Ophthalmol Soc. 1991;89:757-790.

34. Caprioli J. Intraocular pressure fluctuation: an independent risk factor for glaucoma? Arch Ophthalmol. 2007;125(8):1124-1125.
Clinical Ophthalmology

\section{Publish your work in this journal}

Clinical Ophthalmology is an international, peer-reviewed journal covering all subspecialties within ophthalmology. Key topics include: Optometry; Visual science; Pharmacology and drug therapy in eye diseases; Basic Sciences; Primary and Secondary eye care; Patient Safety and Quality of Care Improvements. This journal is indexed on Submit your manuscript here: http://www.dovepress.com/clinical-ophthalmology-journal

\section{Dovepress}

PubMed Central and CAS, and is the official journal of The Society of Clinical Ophthalmology (SCO). The manuscript management system is completely online and includes a very quick and fair peer-review system, which is all easy to use. Visit http://www.dovepress.com/ testimonials.php to read real quotes from published authors. 